\title{
Option Pricing under Linear Autoregressive Dynamics, Heteroskedasticity, and Conditional Leptokurtosis
}

\author{
Christian M. Hafner, Helmut Herwartz *
}

July 1999

\begin{abstract}
Daily returns of financial assets are frequently found to exhibit positive autocorrelation at lag 1 . When specifying a linear AR(1) conditional mean, one may ask how this predictability affects option prices. We investigate the dependence of option prices on autoregressive dynamics under stylized facts of stock returns, i.e., conditional heteroskedasticity, leverage effect, and conditional leptokurtosis. Our analysis covers both a continuous and discrete time framework. The results suggest that a non-zero autoregression coefficient tends to increase the deviation of option prices from Black \& Scholes prices caused by stochastic volatility.
\end{abstract}

Keywords: option pricing, autoregression, heteroskedasticity, GARCH, leverage effect, conditional leptokurtosis

JEL classification: C15, C22, G13

*SFB 373 and Institut für Statistik und Ökonometrie, Humboldt-Universität zu Berlin, Spandauer Str. 1, D-10178 Berlin. Financial support by the Deutsche Forschungsgemeinschaft is gratefully acknowledged. The authors would like to thank Peter Bank and David S. Bates for helpful comments. 


\section{Introduction}

Recent empirical studies of financial markets suggest that there is a substantial body of evidence that documents the predictability of financial asset returns. This was stated by Lo \& Wang (1995), who cite a collection of 30 papers to support the hypothesis of predictability. The term predictability is used as having non-zero autocorrelation of returns. Chapter 2 of Campbell, Lo and MacKinlay (1997) gives a summary of recent empirical evidence for positive autocorrelation at daily and monthly frequency.

A possible economic explanation for such positive autocorrelation could be risk premia that are linked to time-varying second moments. For example, the ARCH-M model of Engle, Lilien and Robins (1987) lets the risk premium be a function of volatility, which is modeled by the ARCH model class. It turned out, however, that autoregressive processes often provide superior results in terms of log-likelihood estimates, as was shown by Hafner and Herwartz (1999) for the German stock market.

Even though the conditional mean does not enter into closed form option pricing solutions such as Black-Scholes, its particular specification may affect the estimates of other relevant parameters such as volatility. This is why it is pertinent to analyse the effects of autoregressive components for option pricing. Since option prices may react sensitively to changes in volatility, a proper specification of the conditional variance and the conditional mean plays a crucial role. In particular, out-of-the-money options with short times to maturity react strongly to volatility changes when measuring this sensitivity in relative terms, i.e., regarding the elasticity of the option price with respect to volatility.

Being the most popular approaches to model volatility clustering, GARCH models as introduced by Engle (1982) and Bollerslev (1986) soon showed their deficiencies: First, stock market volatility is often more affected by negative news than by positive news. This effect, first noted by Black (1976), is known as leverage effect. Many extensions of the standard GARCH model have been suggested that allow for asymmetric impacts of positive and negative news on volatility. Second, in most studies of daily stock returns using GARCH models and conditional normality for the innovations, the rescaled residuals showed excess kurtosis which violates the normality assumption.

In this paper, we analyse the impact of autoregressive dynamics on volatility estimates and on option prices. We separate our analysis to a continuous time and discrete time framework. In continuous time, we derive results for the case of a trend reverting log price and stationary stochastic volatility. Under the assumption that volatility is not priced and log price and volatility are not correlated, one can in the spirit of Hull and White (1987) derive a Taylor expansion of the option price around the expected mean volatility until time to maturity. We analyse the change of the first order term in case of an underlying autoregressive component. We show that under autoregressive dynamics far in- and out-ofthe-money options are valued higher and at-the-money options lower than when neglecting autoregressive dynamics.

Evaluation of analytic formulae in a continuous time framework becomes tedious in case of a negative correlation between the innovations of log price and volatility (i.e., the leverage effect) or leptokurtic innovations. Therefore, we make use of results for discrete time models. In particular, our discrete time framework builds on the work of Duan (1995), whose GARCH option pricing model can be easily extended without losing its interpretation as an economic equilibrium model. We employ the threshold GARCH model of Glosten, Jagannathan and Runkle (1993) and conditional Student-t distributed innovations to model the leverage effect and conditional leptokurtosis, respectively.

In line with our results in the continuous time framework, the results in discrete time suggest that autoregressive dynamics of returns increase the effects caused by stochastic volatility or GARCH. In particular, viewing the deviation of GARCH option prices from Black \& Scholes prices as a function of the moneyness, one obtains the typical U-shape, 
that is, far in- and out-of-the-money options are priced higher and at-the-money options lower. This U-shape becomes more distinct under additional autoregressive dynamics: atthe-money options become even less, far in- and out-of-the-money options even more valuable. This holds for a positive and negative $\operatorname{AR}(1)$ coefficient. In relative terms we find the highest impact of autocorrelation for options out-of-the-money. Conditional leptokurtosis is found to impose an additional smile compared to volatility processes generated under conditional normality. This additional smile appears to be symmetric even in the TGARCH case and is much more pronounced under autoregressive dynamics.

The accordance of our results for the trend reverting stochastic volatility in continuous time and AR/GARCH in discrete time may not be surprising when considering that the diffusion limit of GARCH models is precisely the stochastic volatility process that we assume, see Nelson (1990). Duan (1996) shows that his discrete time option pricing model converges to the Hull and White (1987) stochastic volatility option pricing model if the time interval between observations is decreasing and volatility parameters are appropriately rescaled. However, there is an important difference: Modelling autoregressive dynamics in continuous time with a linear trend reversion process, one can only model negative correlation of returns. To model positive correlation, one has to resort to multivariate processes. Along these lines in a constant volatility framework, Lo and Wang (1995) show that the effects of the autoregression parameter are contrary: negative correlation increases, positive correlation decreases option prices. In discrete time, however, there is no such asymmetry: the sign of the autoregressive parameter is irrelevant, all that matters is its size.

The empirical relevance of our results is illustrated for a set of German stock price series. Specifying an autoregressive model additional to GARCH changed the volatility estimates slightly. This change implied in many cases a substantial deviation of option prices.

The remainder of the paper is organized as follows: Section 2 provides an empirical assessment of the differences of volatility path estimates obtained from alternative modeling assumptions. In Section 3 we first review option pricing under autoregressive dynamics and constant volatility in continuous time, i.e., a trending Ornstein-Uhlenbeck process. Then, we allow for stochastic volatility and develop results for option pricing in a continuous-time framework with a trend reverting log price process. The GARCH option pricing model under linear dynamics is discussed in Sections 4 and 5. The distribution of the independent innovations is assumed to be normal in Section 4 and leptokurtic in Section 5. Section 6 provides a brief summary and concludes. Proofs are provided in Appendix A.

\section{Volatility estimates for alternative models}

The linear AR(1) model with conditionally heteroskedastic errors writes as

$$
y_{t}=\nu+\phi y_{t-1}+\sqrt{h_{t}} \xi_{t},
$$

where $\nu$ and $\phi$ are constant parameters, the innovations $\xi_{t}$ are i.i.d. with mean zero and variance one and the volatility $h_{t}$ is a measurable function of lagged innovations and lagged $h_{t}$. Denoting by $\mathcal{F}_{t}$ the information set up to time $t$, we have the conditional mean $\mu_{t}=$ $\mathrm{E}\left[y_{t} \mid \mathcal{F}_{t-1}\right]=\nu+\phi y_{t-1}$ and the conditional variance $h_{t}=\operatorname{Var}\left[y_{t} \mid \mathcal{F}_{t-1}\right]$.

Specifically, we assume the asymmetric threshold GARCH model

$$
h_{t}=\omega+\left(\left(\alpha+\alpha^{-} I_{\left(\xi_{t-1}<0\right)}\right) \xi_{t-1}^{2}+\beta\right) h_{t-1},
$$

where the common symmetric GARCH model results as a special case for $\alpha^{-}=0$. Dealing with stock returns, one typically obtains positive estimates for $\alpha^{-}$, indicating a stronger impact of negative news on volatility than positive news. Model (2) was proposed by Glosten, Jagannathan and Runkle (1993). A similar threshold model for the conditional standard deviation is due to Zakoian (1994). 
We investigate the daily closing prices $S_{t}$ of 26 German stocks traded at the Frankfurt stock exchange during the period 02/01/1990 to 30/12/1996, providing $n=1753$ observations for each stock. Returns $y_{t}$ are defined as relative price changes, i.e. $y_{t}=$ $\left(S_{t}-S_{t-1}\right) / S_{t-1}$. All return series exhibit strong conditional heteroskedasticity. The ARCHLM test of Engle (1982) rejects the hypothesis of homoskedasticity at all common levels, both for returns and residuals of a linear AR(1) regression.

We estimate GARCH-type models, each with and without an AR(1) conditional mean component. To incorporate an explicit risk premium into the specification, we also estimated models of the GARCH-M type, see Engle, Lilien and Robins (1987). It turned out, however, that unlike for the AR parameter the inclusion of a risk premium did not significantly improve the model fit measured in terms of the log-likelihood for the majority of investigated series. We will thus restrict our considerations to models without risk premium.

QML-inference against the significance of $\phi$ clearly supports the presence of autoregressive dynamics for most return series under study. In seven cases, QML rejects the hypothesis $\phi=0$ when it is accepted by the heteroskedasticity consistent $t$-ratio of White (1980) and the bootstrapped version of a pseudo likelihood ratio statistic, see Herwartz (1998) and Hafner and Herwartz (1999). For the majority of stocks, all test procedures rejected the null hypothesis of no autoregressive dynamics.

\begin{tabular}{lccc|cccc}
\hline \hline \multicolumn{3}{c|}{$\xi_{t} \sim N(0,1)$} & \multicolumn{4}{c}{$\xi_{t} \sim t_{v}(0,1)$} \\
\hline Stock & AR(0) & \multicolumn{2}{c}{ AR(1) } & AR(0) & \multicolumn{2}{c}{ AR(1) } \\
& GARCH & GARCH & TGARCH & GARCH & GARCH & TGARCH & $\hat{v}$ \\
\hline ALLI & 5198.8 & 5210.0 & 5215.9 & 5260.6 & 5265.9 & 5270.3 & 6.6 \\
BASF & 5210.1 & 5211.7 & 5212.2 & 5244.6 & 5245.8 & 5245.8 & 7.2 \\
BAYE & 5238.7 & 5243.0 & 5243.0 & 5304.9 & 5306.2 & 5306.3 & 6.6 \\
BAYH & 5299.1 & 5303.1 & 5307.2 & 5412.7 & 5412.8 & 5414.8 & 4.9 \\
BAYV & 5218.2 & 5218.2 & 5222.1 & 5310.4 & 5310.9 & 5313.6 & 5.1 \\
BMW & 5153.0 & 5153.1 & 5162.6 & 5256.1 & 5259.0 & 5262.7 & 5.1 \\
COMM & 5256.9 & 5259.7 & 5259.9 & 5402.5 & 5402.8 & 5402.9 & 4.8 \\
DAIM & 5093.3 & 5096.7 & 5098.1 & 5156.7 & 5159.3 & 5159.5 & 6.7 \\
DEUT & 5376.4 & 5380.4 & 5380.9 & 5468.1 & 5471.4 & 5471.6 & 6.4 \\
DEGU & 4954.6 & 4954.8 & 4955.9 & 5024.1 & 5024.1 & 5024.6 & 4.9 \\
DRES & 5397.1 & 5397.8 & 5401.9 & 5542.6 & 5542.7 & 5545.0 & 4.9 \\
HENK & 5304.0 & 5309.1 & 5309.1 & 5425.0 & 5425.3 & 5425.4 & 5.1 \\
HOEC & 5074.2 & 5078.4 & 5083.2 & 5147.2 & 5150.6 & 5153.4 & 6.0 \\
KARS & 5138.7 & 5138.9 & 5140.3 & 5199.2 & 5205.3 & 5206.2 & 6.0 \\
LIND & 5437.8 & 5442.0 & 5447.8 & 5567.0 & 5568.5 & 5572.1 & 4.9 \\
MAN & 4884.1 & 4891.0 & 4896.7 & 5013.0 & 5015.3 & 5020.7 & 5.9 \\
MANN & 4867.4 & 4878.4 & 4879.5 & 5008.6 & 5009.0 & 5010.7 & 5.3 \\
MUEN & 5158.8 & 5163.2 & 5184.7 & 5293.8 & 5295.6 & 5312.3 & 3.2 \\
PREU & 5046.2 & 5047.9 & 5055.7 & 5144.4 & 5146.0 & 5149.2 & 5.6 \\
RWE & 5385.7 & 5397.0 & 5400.6 & 5499.4 & 5501.6 & 5503.3 & 5.6 \\
SCHE & 5272.0 & 5272.1 & 5274.9 & 5341.9 & 5342.0 & 5343.7 & 4.9 \\
SIEM & 5461.4 & 5467.1 & 5468.1 & 5565.8 & 5567.0 & 5568.6 & 7.2 \\
THYS & 4898.9 & 4903.8 & 4904.0 & 4961.6 & 4962.6 & 4962.7 & 6.7 \\
VEBA & 5371.5 & 5371.9 & 5372.3 & 5464.6 & 5465.2 & 5465.6 & 6.0 \\
VIAG & 5241.4 & 5243.1 & 5244.0 & 5375.5 & 5377.8 & 5380.3 & 5.2 \\
VOLK & 4773.1 & 4781.3 & 4781.6 & 4839.9 & 4844.2 & 4844.4 & 8.3 \\
\hline \hline
\end{tabular}

Table 1: Log-likelihood estimates of alternative model specifications under conditional normality and conditional leptokurtosis. Estimated degrees of freedom $(\hat{v})$ for AR(1)-TGARCH specification. 
Assuming conditional normality, estimation and diagnostic results can be interpreted in a similar fashion for all investigated return series. First, we find significant ARCH-effects indicated by significant estimates for $\alpha$ using a symmetric $\operatorname{GARCH}(1,1)$ process or $\alpha^{-}$within its asymmetric counterpart, the TGARCH $(1,1)$ model. With respect to the absolute value of parameter estimates we find for the vast majority of investigated return series estimates of $\beta$ close to 0.9 and of $\alpha+\beta$ between 0.960 and 0.999 , indicating the typical high persistence of shocks in volatility. The estimation results obtained for the parameters governing variance dynamics were similar for alternative specifications of the conditional mean equation, i.e. for the $\operatorname{AR}(0)$ versus the $\operatorname{AR}(1)$ model. For all series the $\operatorname{GARCH}(1,1)$ process turns out to remove conditional heteroskedasticity. Applying the ARCH-LM test of order 1 or 5 to the standardized return series the assumption of homoskedasticity is not rejected for all estimated models. Testing standardized innovations against unconditional normality, however, we find that all standardized residual series exhibit excess kurtosis. We refrain from providing all model diagnostics to economize on space. Instead, we refer to Hafner and Herwartz (1999) for further discussion of estimation results obtained under conditional normality. Table 2 reports the estimation results for Allianz.

\begin{tabular}{|c|c|c|c|c|c|c|c|c|}
\hline & \multicolumn{4}{|c|}{$\xi_{t} \sim N(0,1)$} & \multicolumn{4}{|c|}{$\xi_{t} \sim t_{v}(0,1)$} \\
\hline & \multicolumn{2}{|c|}{ GARCH } & \multicolumn{2}{|c|}{ TGARCH } & \multicolumn{2}{|c|}{ GARCH } & \multicolumn{2}{|c|}{ TGARCH } \\
\hline & $\operatorname{AR}(0)$ & $\operatorname{AR}(1)$ & $\operatorname{AR}(0)$ & $\operatorname{AR}(1)$ & $\operatorname{AR}(0)$ & $\operatorname{AR}(1)$ & $\operatorname{AR}(0)$ & $\operatorname{AR}(1)$ \\
\hline$\overline{\hat{\nu}}$ & $\begin{array}{c}5.0 \mathrm{E}-04 \\
(1.88)\end{array}$ & $\begin{array}{c}4.5 \mathrm{E}-04 \\
(1.71)\end{array}$ & $\begin{array}{c}3.2 \mathrm{E}-04 \\
(1.21)\end{array}$ & $\begin{array}{c}2.7 \mathrm{E}-04 \\
(1.01)\end{array}$ & $\begin{array}{c}2.9 \mathrm{E}-04 \\
(1.11)\end{array}$ & $\begin{array}{c}2.6 \mathrm{E}-04 \\
(1.04)\end{array}$ & $\begin{array}{c}1.9 \mathrm{E}-04 \\
(0.74)\end{array}$ & $\begin{array}{c}1.5 \mathrm{E}-04 \\
(0.60)\end{array}$ \\
\hline$\hat{\phi}$ & & $\begin{array}{c}.097 \\
(3.72)\end{array}$ & & $\begin{array}{c}.087 \\
(3.45)\end{array}$ & & $\begin{array}{c}.073 \\
(2.85)\end{array}$ & & $\begin{array}{c}.071 \\
(2.59)\end{array}$ \\
\hline$\hat{\omega}$ & $\begin{array}{c}3.2 \mathrm{E}-06 \\
(1.91)\end{array}$ & $\begin{array}{c}3.2 \mathrm{E}-06 \\
(1.90)\end{array}$ & $\begin{array}{c}2.6 \mathrm{E}-06 \\
(1.84)\end{array}$ & $\begin{array}{c}2.7 \mathrm{E}-06 \\
(1.81)\end{array}$ & $\begin{array}{c}3.1 \mathrm{E}-06 \\
(1.88)\end{array}$ & $\begin{array}{c}3.3 \mathrm{E}-06 \\
(1.90)\end{array}$ & $\begin{array}{c}3.1 \mathrm{E}-06 \\
(1.96)\end{array}$ & $\begin{array}{c}3.3 \mathrm{E}-06 \\
(1.86)\end{array}$ \\
\hline$\hat{\alpha}$ & $\begin{array}{c}.070 \\
(4.52)\end{array}$ & $\begin{array}{c}.075 \\
(4.49)\end{array}$ & $\begin{array}{c}.029 \\
(2.48)\end{array}$ & $\begin{array}{c}.031 \\
(2.41)\end{array}$ & $\begin{array}{c}.066 \\
(3.85)\end{array}$ & $\begin{array}{c}.070 \\
(3.96)\end{array}$ & $\begin{array}{c}.037 \\
(3.04)\end{array}$ & $\begin{array}{c}.039 \\
(2.79)\end{array}$ \\
\hline$\hat{\alpha}^{-}$ & & & $\begin{array}{c}.070 \\
(2.85)\end{array}$ & $\begin{array}{c}.072 \\
(3.06)\end{array}$ & & & $\begin{array}{c}.061 \\
(2.64)\end{array}$ & $\begin{array}{c}.065 \\
(2.35)\end{array}$ \\
\hline$\hat{\beta}$ & $\begin{array}{c}.914 \\
(49.4)\end{array}$ & $\begin{array}{c}.909 \\
(46.6)\end{array}$ & $\begin{array}{c}.923 \\
(56.1)\end{array}$ & $\begin{array}{c}.920 \\
(53.6)\end{array}$ & $\begin{array}{c}.916 \\
(39.2)\end{array}$ & $\begin{array}{c}.911 \\
(37.4)\end{array}$ & $\begin{array}{c}.917 \\
(41.9)\end{array}$ & $\begin{array}{c}.912 \\
(34.3)\end{array}$ \\
\hline$\hat{v}$ & & & & & $\begin{array}{c}6.18 \\
(6.52)\end{array}$ & $\begin{array}{c}6.38 \\
(6.20)\end{array}$ & $\begin{array}{c}6.48 \\
(6.30)\end{array}$ & $\begin{array}{c}6.66 \\
(6.11)\end{array}$ \\
\hline
\end{tabular}

Table 2: Estimation results for Allianz returns. QML $t$-ratios in parantheses.

Taking conditional leptokurtosis into account we estimated all processes considered assuming the innovations $\xi_{t}$ to be generated by a standardized Student-t distribution. For the GARCH model specified with and without an autoregressive component and for the AR(1)TGARCH processes Table 1 displays the obtained maximum values of the log-likelihood function obtained under normality and Student- $t$ innovations, respectively. In addition, the estimated degrees of freedom obtained for the AR(1)-TGARCH Student-t model are reported. Obviously, the assumption of leptokurtic innovations provides considerable improvements of the empirical models specified under normality in terms of the log-likelihood values. For the $\mathrm{AR}(0)-\mathrm{GARCH}$ we obtain log-likelihood improvements between 34.5 for BASF and 145.6 for Commerzbank. The significance of these improvements can also be inferred from the estimated degrees of freedom $\hat{v}$ for the Student-t model being in most cases close to 6 . Regarding the corresponding standard deviations it would become obvious that for all investigated series the estimated Student-t distributions differ significantly from a normal approximation $v \approx 30$.

Given a specific return series it turned out that with respect to the estimation of param- 


\begin{tabular}{lccc|ccc|ccc}
\hline \hline & \multicolumn{3}{c}{ AR(0) } & \multicolumn{3}{c}{ GARCH } & \multicolumn{3}{c}{ TGARCH } \\
\hline Stock & MAD & Min & Max & MAD & Min & Max & MAD & Min & Max \\
\hline ALLI & 11.8 & -38.4 & 41.4 & 2.58 & -8.01 & 9.78 & 2.37 & -8.03 & 10.4 \\
BASF & 4.26 & -15.7 & 16.6 & 0.61 & -2.41 & 3.84 & 0.64 & -2.39 & 4.02 \\
BAYE & 7.05 & -24.6 & 36.6 & 0.23 & -1.15 & 0.86 & 0.26 & -0.66 & 1.03 \\
BAYH & 7.00 & -34.0 & 25.8 & 1.41 & -5.63 & 5.87 & 1.36 & -4.70 & 5.47 \\
BAYV & 0.23 & -0.89 & 1.36 & 1.50 & -6.33 & 6.38 & 1.55 & -7.08 & 6.92 \\
BMW & 0.91 & -2.92 & 4.31 & 2.74 & -9.52 & 12.6 & 2.73 & -10.1 & 12.7 \\
COMM & 5.50 & -20.3 & 21.7 & 0.33 & -1.09 & 1.59 & 0.35 & -1.22 & 1.73 \\
DAIM & 6.74 & -20.6 & 31.2 & 0.66 & -2.62 & 1.96 & 0.65 & -3.19 & 2.23 \\
DEUT & 7.91 & -37.0 & 34.7 & 0.54 & -2.24 & 3.01 & 1.09 & -6.15 & 5.01 \\
DEGU & 1.60 & -7.39 & 6.24 & 0.88 & -2.90 & 5.73 & 0.98 & -3.01 & 5.77 \\
DRES & 2.87 & -10.2 & 15.5 & 1.82 & -6.34 & 7.65 & 1.70 & -6.34 & 7.13 \\
HENK & 7.49 & -30.6 & 18.5 & 0.31 & -0.69 & 0.59 & 0.14 & -0.45 & 1.10 \\
HOEC & 7.42 & -32.5 & 34.6 & 1.69 & -9.27 & 10.1 & 1.78 & -9.29 & 9.04 \\
KARS & 1.99 & -11.0 & 7.26 & 0.66 & -4.39 & 3.23 & 0.62 & -4.47 & 3.46 \\
LIND & 6.58 & -35.2 & 42.8 & 1.85 & -15.6 & 9.06 & 1.62 & -15.1 & 8.13 \\
MAN & 8.78 & -46.0 & 54.1 & 1.43 & -10.5 & 18.0 & 1.41 & -8.09 & 14.6 \\
MANN & 10.3 & -40.2 & 53.1 & 1.91 & -13.0 & 13.5 & 1.40 & -8.29 & 10.6 \\
MUEN & 6.98 & -27.2 & 25.1 & 5.78 & -29.9 & 29.8 & 4.03 & -27.7 & 16.3 \\
PREU & 6.07 & -17.6 & 31.4 & 1.44 & -5.10 & 4.98 & 1.51 & -5.87 & 6.61 \\
RWE & 11.7 & -33.9 & 71.4 & 2.52 & -9.74 & 15.3 & 1.80 & -6.07 & 8.89 \\
SCHE & 0.83 & -2.42 & 3.50 & 1.59 & -8.04 & 8.58 & 1.60 & -7.96 & 8.80 \\
SIEM & 9.15 & -34.9 & 36.8 & 0.36 & -1.83 & 1.93 & 0.47 & -2.45 & 2.17 \\
THYS & 8.11 & -39.5 & 25.7 & 0.28 & -1.35 & 1.17 & 0.28 & -0.92 & 1.44 \\
VEBA & 1.88 & -8.24 & 7.33 & 0.84 & -3.29 & 4.71 & 0.87 & -3.48 & 4.83 \\
VIAG & 4.46 & -19.3 & 24.2 & 1.07 & -3.65 & 3.79 & 0.93 & -3.55 & 4.23 \\
VOLK & 9.53 & -48.4 & 31.0 & 0.40 & -1.83 & 2.35 & 0.39 & -1.80 & 1.74 \\
\hline \hline
\end{tabular}

Table 3: Mean absolute deviation (MAD), minimum and maximum of the percentage differences between volatility estimates obtained under conditional normality.

eters governing conditional variances only negligible differences are obtained for alternative specifications of the conditional mean equation. Obviously this result mirrors the typically low degree of explanation provided by an autoregressive model of order one applied to return series. For almost all data sets under study the estimated AR(1) coefficients are positive and less than 0.1. The latter result is invariant with respect to the choice of a normal compared to a leptokurtic distribution of innovations $\xi_{t}$. For most series, however, estimated AR(1) parameters are somewhat smaller assuming Student-t compared to normally distributed innovations. The evidence in favour of autoregressive dynamics is mitigated to some extent assuming Student-t innovations. As a possible device to test for autoregressive dynamics convenient likelihood ratio statistics can also be obtained from Table 1. For example, assuming a $\operatorname{GARCH}(1,1)$ variance process with normally distributed innovations, we find significant autoregressive dynamics with significance level $5 \%$ for 16 of 26 return series. Taking the same formal test criterion for the $\operatorname{GARCH}(1,1)$ model under Student-t distributed innovations we obtain autoregressive dynamics for only 9 return series investigated.

Regarding estimated parameters $\alpha, \alpha^{-}$and $\beta$ governing variance dynamics we neither obtain major differences for the two alternative assumptions made for the distribution generating $\xi_{t}$, nor for the specification of $\operatorname{AR}(1)$ or $\operatorname{AR}(0)$. Still, the estimated volatility paths may differ substantially. Volatility depends on excess returns in the $\operatorname{AR}(0)$ model and on errors in the AR(1) model. Under conditional normality, the innovations in volatility are 


\begin{tabular}{lccc|ccc|ccc}
\hline \hline & \multicolumn{3}{c}{ AR(0) } & \multicolumn{3}{c}{ GARCH } & \multicolumn{3}{c}{ TGARCH } \\
\hline Stock & MAD & Min & Max & MAD & Min & Max & MAD & Min & Max \\
\hline ALLI & 10.1 & -31.6 & 40.9 & 2.05 & -6.39 & 7.59 & 2.03 & -6.80 & 8.95 \\
BASF & 4.41 & -15.0 & 18.8 & 0.12 & -0.46 & 0.67 & 0.10 & -0.45 & 0.56 \\
BAYE & 5.05 & -14.2 & 27.7 & 0.42 & -2.39 & 1.34 & 0.37 & -2.00 & 1.31 \\
BAYH & 1.82 & -7.37 & 8.12 & 1.06 & -4.20 & 4.86 & 1.18 & -5.03 & 6.23 \\
BAYV & 3.35 & -11.8 & 15.6 & 1.10 & -4.20 & 5.68 & 1.11 & -3.61 & 4.15 \\
BMW & 7.94 & -23.8 & 33.6 & 1.96 & -6.65 & 7.25 & 1.87 & -6.45 & 4.93 \\
COMM & 2.70 & -8.04 & 14.2 & 0.25 & -1.08 & 1.13 & 0.26 & -1.00 & 1.24 \\
DAIM & 6.57 & -22.4 & 24.2 & 0.36 & -1.51 & 0.92 & 0.33 & -1.51 & 0.88 \\
DEUT & 8.06 & -26.7 & 35.7 & 0.28 & -0.99 & 1.32 & 0.33 & -1.15 & 1.46 \\
DEGU & 0.49 & -1.64 & 2.12 & 0.57 & -2.11 & 2.78 & 0.61 & -2.59 & 2.91 \\
DRES & 1.57 & -4.55 & 8.90 & 1.42 & -4.98 & 5.18 & 1.39 & -4.84 & 5.23 \\
HENK & 3.24 & -9.35 & 23.0 & 0.07 & -0.31 & 0.40 & 0.11 & -0.43 & 0.63 \\
HOEC & 8.39 & -28.3 & 45.9 & 1.23 & -6.41 & 5.55 & 1.31 & -7.04 & 5.32 \\
KARS & 10.9 & -44.5 & 85.5 & 2.71 & -25.1 & 23.4 & 0.92 & -5.53 & 8.27 \\
LIND & 5.30 & -20.1 & 23.5 & 2.01 & -12.1 & 7.40 & 1.93 & -11.8 & 6.71 \\
MAN & 7.08 & -24.0 & 28.1 & 2.11 & -9.24 & 12.1 & 2.23 & -8.93 & 11.5 \\
MANN & 3.10 & -9.89 & 15.7 & 0.79 & -3.02 & 4.18 & 0.86 & -3.81 & 4.36 \\
MUEN & 6.65 & -25.5 & 15.6 & 4.77 & -22.3 & 12.4 & 5.10 & -26.6 & 12.8 \\
PREU & 5.58 & -19.6 & 21.6 & 1.23 & -4.58 & 2.94 & 1.30 & -5.06 & 3.22 \\
RWE & 6.94 & -18.2 & 39.0 & 1.05 & -4.03 & 4.43 & 1.14 & -4.53 & 5.15 \\
SCHE & 1.23 & -4.27 & 3.97 & 1.32 & -6.05 & 5.72 & 1.34 & -6.27 & 5.92 \\
SIEM & 5.04 & -18.4 & 17.4 & 0.75 & -4.12 & 2.88 & 0.83 & -3.92 & 3.80 \\
THYS & 4.41 & -18.8 & 13.9 & 0.17 & -0.91 & 0.52 & 0.16 & -0.94 & 0.50 \\
VEBA & 3.48 & -11.9 & 18.0 & 0.58 & -1.89 & 3.32 & 0.67 & -2.04 & 3.63 \\
VIAG & 7.35 & -34.5 & 36.8 & 1.33 & -4.75 & 7.94 & 1.48 & -5.53 & 7.98 \\
VOLK & 8.32 & -36.5 & 29.6 & 0.28 & -1.21 & 1.33 & 0.27 & -1.12 & 1.09 \\
\hline \hline
\end{tabular}

Table 4: Mean absolute deviation (MAD), minimum and maximum of the percentage differences between volatility estimates obtained under conditional leptokurtosis.

chi-square, in the Student-t model F-distributed after appropriate rescaling.

To shed some light on the dependence of volatility estimates obtained from alternative model specifications we generated series of the form $V_{1} / V_{2}-1$, where $V_{1}$ and $V_{2}$ are volatility processes estimated under specific assumptions. In particular, we provide a comparison of $\operatorname{GARCH}(1,1)$ volatility estimates with $\operatorname{TGARCH}(1,1)$ counterparts in order to report on the empirical magnitude of the prominent leverage effect. Similarly, we also provide a comparison for volatility processes derived from the $\operatorname{AR}(0)$ and $\operatorname{AR}(1)$ model. The latter comparison is performed assuming both a $\operatorname{GARCH}(1,1)$ and $\operatorname{TGARCH}(1,1)$ specification. Tables 3 and 4 provide analogous measures obtained from the normal model and the Studentt model, respectively. In particular, for relative differences $V_{1} / V_{2}-1$ we provide the mean absolute deviation (MAD) and the observed maximum and minimum values.

Obviously, generalizing the symmetric $\operatorname{GARCH}(1,1)$ variance towards an asymmetric impact of lagged innovations on current volatility substantially affects the estimated volatility path. Assuming normally distributed innovations, relative differences as described above show for 17 of 26 series mean average deviations of at least $5 \%$. A similar result is obtained for processes specified with Student-t distributed innovations. Specific observed differences vary between $-46.0 \%$ and $+71.4 \%$ for the volatility paths estimated under normality. Compared to the empirical importance of the leverage effect, inclusion and exclusion of autoregressive dynamics is of less importance. Under normality we obtain mean absolute differences 


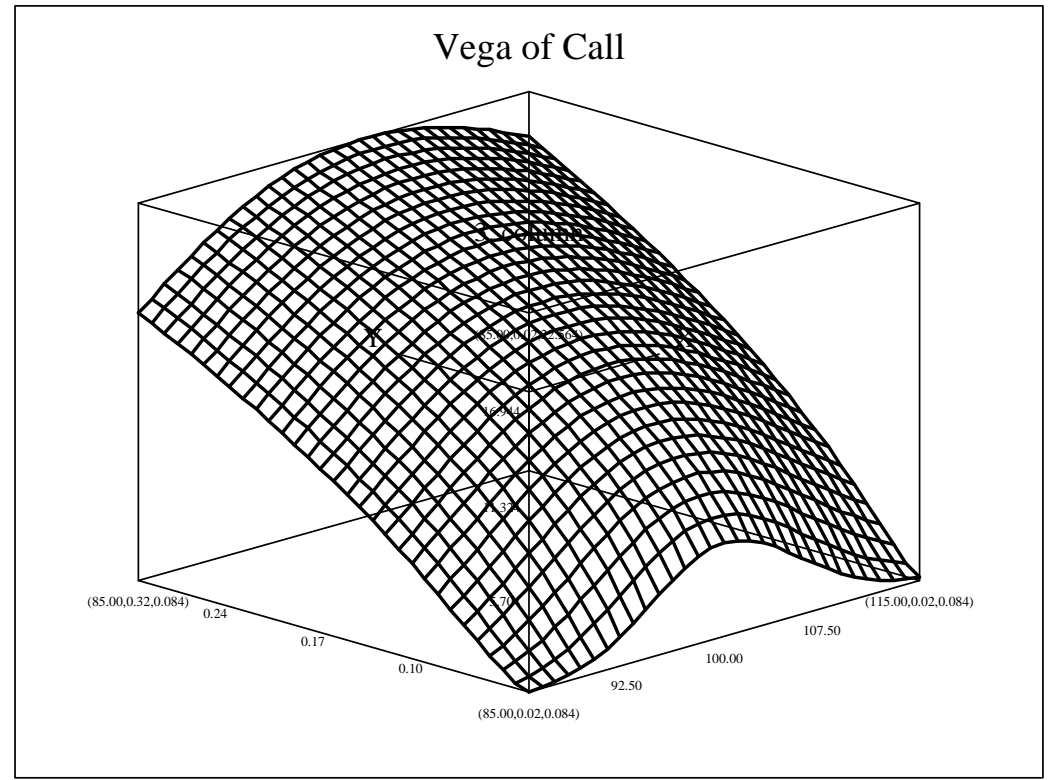

Figure 1: The Black and Scholes Vega of a Call option as given in (4) with time to maturity (left axis) from 0.02 to 0.32 and stock price (right axis) from 85 to 115 . The exercise price was fixed at 100, the volatility parameter at 0.4 and the interest rate at zero.

between volatility estimates obtained from the $\mathrm{AR}(1)$ and $\mathrm{AR}(0)$ model alternatively to differ more than $1 \%$ on average for 14 series considered. The latter result holds for the $\operatorname{GARCH}(1,1)$ and $\operatorname{TGARCH}(1,1)$ and also for the $\operatorname{GARCH}(1,1)$ model estimated under the assumption of Student-t distributed innovations. In specific periods, however, relative differences of volatility measures may differ substantially with respect to the specification of the conditional mean equation. The estimated minimum and maximum statistics for relative volatility differences between the $\mathrm{AR}(0)$ and $\mathrm{AR}(1)$ model vary between $-29.9 \%$ and $29.8 \%$ percent.

The average impact of autoregressive dynamics on volatility estimates might seem negligible, but considering financial derivatives, a small percent difference in volatility may imply a large percent difference in the option price. This occurs if the elasticity of the option price $C$ with respect to volatility $\sigma$, i.e.

$$
\eta=\frac{\partial C}{\partial \sigma} \frac{\sigma}{C}=\mathcal{V} \frac{\sigma}{C}
$$

is large, where $\mathcal{V}$ is sometimes referred to as the option's Vega. Here, $\sigma$ may be either a constant parameter as in the Black and Scholes model, or the time-varying conditional volatility $\sqrt{h_{t}}$ in the GARCH framework.

To have an idea how Vega and $\eta$ look like in the Black and Scholes world, they are plotted in Figures 1 and 2 as a function of the stock price $\mathrm{S}$ and time to maturity $\tau=T-t$.

The formula for the Vega of a call option is

$$
\mathcal{V}=S_{t} \sqrt{\tau} \psi\left(d_{1}\right)
$$




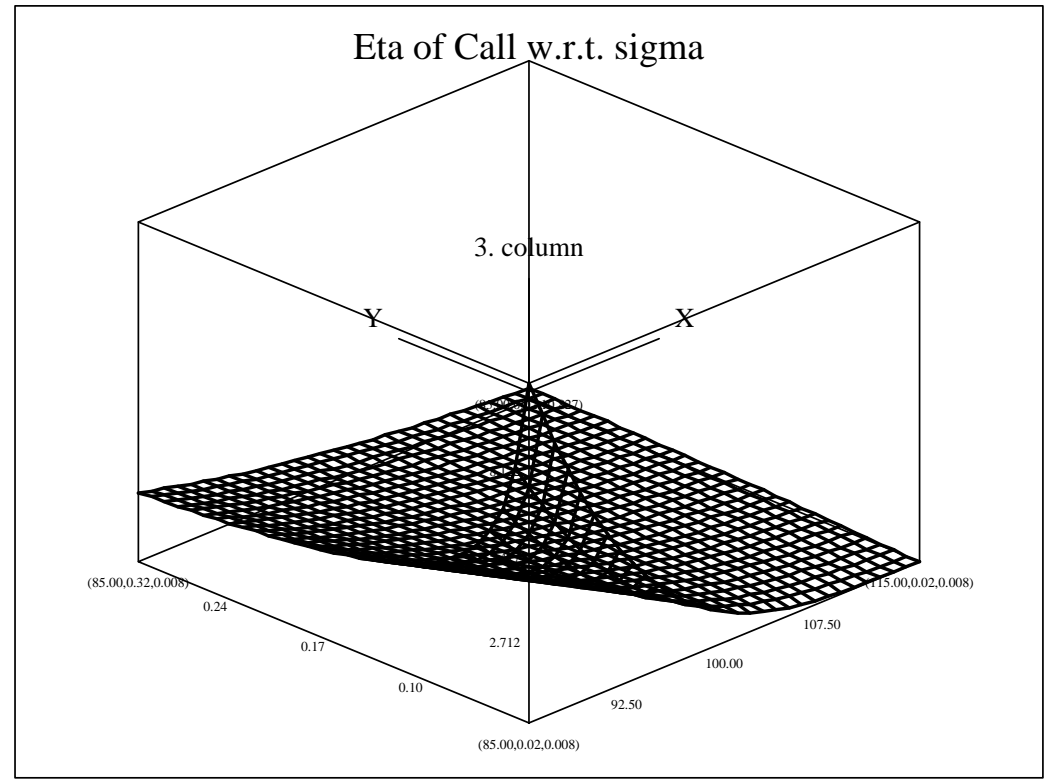

Figure 2: The Black and Scholes elasticity of a Call option with respect to volatility as given in (3) with time to maturity (left axis) from 0.02 to 0.32 and stock price (right axis) from 85 to 115 . The exercise price was fixed at 100, the volatility parameter at 0.4 and the interest rate at zero.

with

$$
d_{1}\left(\sigma^{2}\right)=\frac{\log \left(S_{t} / K\right)+\left(r+\sigma^{2} / 2\right) \tau}{\sigma \sqrt{\tau}},
$$

where $K$ is the exercise price, $r$ the constant risk-free interest rate and $\psi$ the standard normal density function. The Black and Scholes (1973) formula for the price of a call option is

$$
C_{B S}\left(S_{t}, t, \sigma^{2}\right)=S_{t} \Phi\left(d_{1}\right)-K e^{-r \tau} \Phi\left(d_{2}\right)
$$

with $\Phi$ denoting the standard normal distribution function and $d_{2}=d_{1}-\sigma \sqrt{\tau}$. Unlike for the constant parameters $K, T$ and $r$, the dependence of $C_{B S}$ on $\sigma$ is made explicit since we will consider time-varying volatility in the following sections.

The plot of Vega in Figure 1 suggests that in absolute terms options at the money with long maturities react most sensitively to changes in volatility. On the other hand, measuring the effects of changes in volatility in relative terms, one realizes from Figure 2 that options reacting most sensitively are out-of-the-money with short maturities. For our choice of the parameters in this example, $\eta$ attains a level of 10 at a moneyness of 0.85 and time to maturity of 0.02 years, approximately one week. In other words, a one percent change of volatility over a small time interval implies a ten percent change of the Black and Scholes option price. 


\section{Linear dynamics in continuous time}

In a continuous time framework, Lo and Wang (1995) considered the case of a trending Ornstein Uhlenbeck (OU) process for the logarithm of the stock price process $S(t)$, i.e.

$$
d \log S_{t}=\left(-\gamma\left(\log S_{t}-\mu t\right)+\mu\right) d t+\sigma d W_{t},
$$

where $W_{t}$ is the standard Wiener process, $\mu$ and $\sigma$ the trend and volatility parameters, respectively, and $\gamma$ the rate at which the stock price is pulled back to its deterministic trend. Returns over time intervals of length $\Delta$ can be defined as

$$
y_{t}(\Delta)=\log S_{t}-\log S_{t-\Delta}
$$

for which the second order moments can be calculated as

$$
\operatorname{Var}\left(y_{t}(\Delta)\right)=\frac{\sigma^{2}}{\gamma}(1-\exp (-\gamma \Delta))
$$

with $\gamma>0$, and

$$
\rho_{1}(\Delta)=\operatorname{Corr}\left(y_{t}(\Delta), y_{t+\Delta}(\Delta)\right)=-\frac{1}{2}(1-\exp (-\gamma \Delta)) .
$$

Even though the returns exhibit time dependence and may be predicted using the negative first order autocorrelations given by (7), the Black and Scholes (1973) formula remains valid since we are in a complete market framework and the Black and Scholes (BS) formula does not depend on the drift.

However, the difference here to the standard BS model is that the parameter estimates used to plug into the BS formula change. In particular, the volatility parameter is adjusted to a higher level. To see this, note that the empirical variance of a return process observed over $n$ time intervals of length $\Delta$ is

$$
s^{2}(y(\Delta))=\frac{1}{n} \sum_{t=1}^{n}\left(y_{t}(\Delta)-\bar{y}(\Delta)\right)^{2}
$$

with $\bar{y}(\Delta)=\frac{1}{n} \sum_{t=1}^{n} y_{t}(\Delta)$, and that empirical moments should match theoretical moments,

$$
\frac{\sigma^{2}}{\gamma}(1-\exp (-\gamma \Delta))=s^{2}(y(\Delta))
$$

From this follows that

$$
\sigma^{2}=\frac{s^{2}(y(\Delta))}{\Delta} \frac{\log \left(1+2 \rho_{1}(\Delta)\right)}{2 \rho_{1}(\Delta)}
$$

where the first term would be used to estimate the volatility parameter in a BS framework. The second term, which is larger than one, adjusts this standard BS volatility to a higher level. The option price is then obtained from the BS formula (6) by plugging in the adjusted volatility $\sigma^{2}$ given in (8). Since option prices depend positively on volatility, negative autocorrelation implies an increase of option prices in this continuous-time, complete market framework. It should be emphasized that we deal with the consequences of model misspecification: Erroneously neglecting autoregressive dynamics underestimates the volatility parameter and, thus, option prices.

Lo and Wang (1995) also consider more general, multivariate processes that allow for positive return autocorrelation. They find that option prices decrease when there is positive autocorrelation. This, however, is a peculiarity of the trending OU-process and does not hold in general. 
In this complete market framework, the only difference between the Black \& Scholes and Lo \& Wang models is the different estimation of the diffusion parameter $\sigma$. Thus, once linear dynamics are identified for a given return series, estimation of the diffusion coefficient should be adjusted accordingly. All that matters here is the correct estimation of the diffusion coefficient.

The diffusion coefficient here is a constant parameter which remains unchanged under risk neutralization. In a more general framework where volatility is stochastic, the volatility dynamics will in general change after risk neutralizing the process. In particular, parameters that determine the drift function may appear in the volatility process. In the following we discuss situations where autoregressive dynamics have non-trivial effects on option prices. By non-trivial we mean that even when the agent correctly employs the unconditional volatility of the risk neutralized process, the change of the option price caused by autoregressive dynamics depends on the moneyness and on the time to maturity.

Consider the following model:

$$
\begin{aligned}
d \log S_{t} & =\left(-\gamma\left(\log S_{t}-\mu t\right)+\mu\right) d t+\sigma_{t} d W_{1, t} \\
d \sigma_{t}^{2} & =\left(\varpi-\theta \sigma_{t}^{2}\right) d t+\delta \sigma_{t}^{2} d W_{2, t}
\end{aligned}
$$

where $W_{1, t}$ and $W_{2, t}$ are two independent Wiener processes and $\varpi, \theta$ and $\delta$ are constant parameters. Volatility $\sigma_{t}$ now is the solution of a stochastic differential equation. By Theorem 2.3 of Nelson (1990), there is a strictly stationary solution to (10) with an inverse Gamma stationary distribution if $2 \theta+\delta^{2}>0$ and $\varpi>0$, which we will assume in the following. Furthermore, we assume $\delta^{2}<2 \theta$, which ensures the existence of fourth order moments of $\log S_{t}$, see Drost and Werker (1996).

Hull and White (1987) only consider the case $\varpi=0$, which implies a less appealing nonstationary volatility process. The empirical support for nonstationarity of volatility, however, is rather weak. For example, volatility of financial return series estimated using GARCH or discrete time stochastic volatility typically satisfies the condition for strict stationarity. Also, a nice property of the diffusion system (9)-(10) is that standard time series models in discrete time such as GARCH have the diffusion limit (10) and may serve as approximations.

Because there is no traded asset which is perfectly correlated with volatility, the market is incomplete. Option valuation is no longer preference-free and one has to make assumptions concerning the pricing of volatility risk. Since the two sources of risk in our model are uncorrelated, we assume that volatility risk is not priced. This corresponds to the choice of the minimal martingale measure of Föllmer and Sondermann (1986) and Föllmer and Schweizer (1991), a consequence of which is that every nontradable asset is not priced, see also Theorem 3.1 of Hofmann, Platen and Schweizer (1992). Under this assumption, the option price is

$$
\begin{aligned}
C\left(S_{t}, t\right) & =\mathrm{E}\left[C_{B S}\left(S_{t}, t, \bar{V}_{\tau}\right) \mid \mathcal{F}_{t}\right] \\
& =\int_{\mathbb{R}_{+}} C_{B S}\left(S_{t}, t, \bar{V}_{\tau}\right) d H\left(\bar{V}_{\tau} \mid \sigma_{t}\right)
\end{aligned}
$$

with $\bar{V}_{\tau}$ denoting the mean volatility over time to maturity,

$$
\bar{V}_{\tau}=\frac{1}{\tau} \int_{t}^{T} \sigma_{s}^{2} d s
$$

with conditional distribution function $H$. In words, the option price is the expected option price of the Black-Scholes model, where the expectation is taken with respect to the mean volatility until time of maturity. No analytic expression is known for $H$, but following the approach of Hull and White (1987) the moments of $\bar{V}_{\tau}$ can be given, allowing a Taylor series approximation of $C$ around the mean of $\bar{V}_{\tau}$. To simplify the analytic expressions, we assume 
that $\sigma_{t}^{2}$ is drawn from its stationary distribution, allowing to consider the unconditional moments of $\bar{V}_{\tau}$. It can be justified by a sufficiently long time to maturity, so that by the stationarity of volatility the law of $\bar{V}_{\tau}$ will be less affected by the starting value $\sigma_{t}^{2}$. The first two moments of $\bar{V}_{\tau}$ are provided in the following proposition.

\section{Proposition 1}

$$
\begin{aligned}
E\left[\bar{V}_{\tau}\right] & =\frac{\varpi}{\theta}=: v \\
\operatorname{Var}\left[\bar{V}_{\tau}\right] & =\frac{2 v^{2} \delta^{2}}{\theta-\delta^{2} / 2} \frac{\theta \tau+e^{-\theta \tau}-1}{\theta^{2} \tau^{2}}
\end{aligned}
$$

A Taylor series approximation of (11) around $v$, the mean of $\bar{V}_{\tau}$, yields

$$
C\left(S_{t}, t\right)=C_{B S}\left(S_{t}, t, v\right)+\frac{1}{2} \frac{\partial^{2} C_{B S}}{\partial \bar{V}_{\tau}^{2}}(v) \operatorname{Var}\left[\bar{V}_{\tau}\right]+\frac{1}{6} \frac{\partial^{3} C_{B S}}{\partial \bar{V}_{\tau}^{3}}(v) \operatorname{Skew}\left(\bar{V}_{\tau}\right)+\ldots
$$

with

$$
\frac{\partial^{2} C_{B S}}{\partial \bar{V}_{\tau}^{2}}(v)=\frac{S_{t} \psi\left(d_{1}\right)\left(d_{1} d_{2}-1\right) \sqrt{\tau}}{4 v^{3 / 2}}
$$

where $d_{1}=d_{1}(v)$ is given in (5) and $d_{2}=d_{1}(v)-\sqrt{v \tau}$.

Plugging (14) and (12) into (13) and ignoring terms of third and higher order ${ }^{1}$, we obtain

$$
C\left(S_{t}, t\right)=C_{B S}\left(S_{t}, t, v\right)+A\left(S_{t}, K, \tau, v\right) B\left(\delta^{2}, \theta, \tau\right)
$$

with

and

$$
A\left(S_{t}, K, \tau, v\right)=S_{t} \psi\left(d_{1}\right)\left(d_{1} d_{2}-1\right) \sqrt{v \tau}
$$

$$
B\left(\delta^{2}, \theta, \tau\right)=\frac{\delta^{2}}{4\left(\theta-\delta^{2} / 2\right)} \frac{\theta \tau+e^{-\theta \tau}-1}{\theta^{2} \tau^{2}} .
$$

Note that $B>0$. The term $A$, viewed as a function of the moneyness $S_{t} / K$, produces the typical U-shape of option prices under stochastic volatility compared with BS prices.

In the following we perform a comparative statics analysis of the terms $A$ and $B$ with respect to the volatility parameters $\varpi, \theta$ and $\delta$. These parameters are affected when autoregressive dynamics are present. As in the Lo and Wang model, erroneously neglecting autoregressive dynamics leads to different parameter estimates. First, we need the signs of the partial derivatives of $A$ and $B$.

\section{Proposition 2}

$$
\frac{\partial A}{\partial \sqrt{v}}\left\{\begin{array}{cl}
<0 & \text { at-the-money, if } \quad r \tau<1 / 2 \sqrt{v \tau+4(1+\sqrt{2+v \tau})} \\
>0 & \text { far in- and out-of-the-money. }
\end{array}\right.
$$

Note that the condition $r \tau<1 / 2 \sqrt{v \tau+4(1+\sqrt{2+v \tau})}$ will hold unless the interest rate is unusually high.

To evaluate how the parameters change we make use of the fact that discrete time GARCH models may serve as diffusion approximations. In Appendix B, we show that

\footnotetext{
${ }^{1}$ Hull and White (1987) note that the Taylor series approximation converges fast if $\delta^{2} \tau$ is not too large.
} 
GARCH parameter estimates, ignoring the underlying AR(1) dynamics, imply an increase of the diffusion parameters $\delta$ and $\varpi$, whereas $\theta$ remains relatively stable. The total differential $d B=\left(\partial B / \partial \delta^{2}\right) d \delta^{2}+(\partial B / \partial \theta) d \theta$ is shown to be positive for various parameter constellations.

The conclusion is that the U-shape caused by stochastic volatility becomes more pronounced in the presence of a trend reversion process. That is, at-the-money options become even less, far-in- and out-of-the-money options even more valuable than without linear dynamics.

\section{Linear dynamics and GARCH volatility}

In this section, we investigate the implications of $\operatorname{AR}(1)$ dynamics in discrete time with conditional heteroskedasticity for option pricing. As option pricing under stochastic volatility has been a main issue of finance theory at least since Hull and White (1987), the combination of option pricing theory with econometrics and in particular the ARCH literature is much younger. The theoretical difficulty arises from the fact that under stochastic volatility the market is in general incomplete. This implies a multitude of equivalent martingale measures and, thus, there is a multitude of no-arbitrage prices.

Recently, Duan (1995) introduced the GARCH option pricing model by generalizing the traditional risk neutral valuation methodology to the case of conditional heteroskedasticity. This so-called locally risk-neutral valuation relationship (LRNVR) has as its essential feature the equivalence of the conditional variances under the data generating probability measure $P$ and the equivalent martingale measure $Q$. The definition further requires that under $Q$ returns are conditionally normally distributed with conditional expectation equal to the riskfree interest rate. For some commonly used assumptions concerning utility functions and distributions of changes of consumption, Duan shows that a representative agent maximizes his expected utility using the LRNVR measure $Q$. This theory holds for general specifications of the conditional mean $\mu_{t}$ and the conditional variance $h_{t}$. Hence, we may employ this approach to investigate the joint effects of autoregressive dynamics and GARCH volatility on option prices. In particular, it will be of interest to compare the impact of positive and negative autoregression parameters on option prices.

A further appealing property of Duan's approach is that the weak limit of his martingale measure is the minimal martingale measure of Föllmer and Sondermann (1986) and Föllmer and Schweizer (1991), see Duan (1996). Many bivariate diffusion models, such as the Hull and White (1987) model, may be recovered from Duan's model. A general result is that volatility risk is not priced unless volatility and stock price changes are correlated, as in the TGARCH case.

Letting the conditional mean $\mu_{t}$ and conditional variance $h_{t}$ be measurable functions with respect to the information set, the general model under the data generating probability measure $P$ is

$$
\begin{aligned}
y_{t} & =\mu_{t}+\sqrt{h_{t}} \xi_{t} \\
\xi_{t} & \sim i . i . d . N(0,1) \\
h_{t} & =f\left(h_{s}, \xi_{s} ;-\infty<s<t ; \theta\right) .
\end{aligned}
$$

where $f$ is a parametric function with parameter vector $\theta$. Under the LRNVR measure $Q$, the $P$-innovation $\xi_{t}$ is shifted by some $\mathcal{F}_{t-1}$-measurable function $\lambda_{t}$ such that the resulting conditional expectation of returns is equal to the risk-free interest rate, which is assumed to be constant. A slightly more general definition was proposed by Duan (1999), who defines returns to be a Box-Cox transformation of $S_{t} / S_{t-1}$, including logarithmic returns. Since we will extend the distributional assumption in (17) to fat-tailed distributions such as the Student-t, we refrain from using the definition of logarithmic returns, because in that case no moments of $S_{t} / S_{t-1}$ exist. 
Under the equivalent martingale measure $Q$, the model writes as

$$
\begin{aligned}
y_{t} & =\mu_{t}+\sqrt{h_{t}}\left(Z_{t}-\lambda_{t}\right) \\
Z_{t} & \sim i . i . d . N(0,1) \\
h_{t} & =f\left(h_{s}, \xi_{s} ;-\infty<s<t ; \theta\right) \\
\xi_{t} & =Z_{t}-\lambda_{t} \\
\lambda_{t} & =\frac{\mu_{t}-r}{\sqrt{h_{t}}} .
\end{aligned}
$$

Obviously, we have $\operatorname{Var}^{P}\left(y_{t} \mid \mathcal{F}_{t-1}\right)=\operatorname{Var}^{Q}\left(y_{t} \mid \mathcal{F}_{t-1}\right)$ and $\mathrm{E}^{Q}\left[y_{t} \mid \mathcal{F}_{t-1}\right]=r$. Note that the system (19) to (23) is general in the sense that neither the conditional mean $\mu_{t}$ nor the conditional variance $h_{t}$ are specified. For $h_{t}$, Duan $(1995)$ used the $\operatorname{GARCH}(1,1)$ model. To incorporate the leverage effect, we employ the threshold GARCH model as in Härdle and Hafner (1999).

For the conditional mean $\mu_{t}$, Duan (1995) and Härdle and Hafner (1999) used the GARCH-M model, i.e., $\mu_{t}=\nu+\lambda \sqrt{h_{t}}$. A theoretical justification for such a choice may come from the finance literature, where intertemporal versions of the capital asset pricing model (CAPM) have been established that provide a link between the CAPM and a multivariate GARCH-M model, see Bollerslev, Engle and Wooldridge (1988) and Hafner and Herwartz (1998). However, there are important differences: First, the CAPM suggests a linear dependence of the stock index risk premium on the variance instead of the standard deviation, and second, the risk premia of individual stocks depend linearly on their covariances with the market portfolio.

In a bivariate framework, Hafner and Herwartz (1998) show that additional to the CAPM risk premium a vector autoregressive component of order one, VAR(1), substantially improves the model fit for most German stocks. As mentioned above, Hafner and Herwartz (1999) show in a univariate framework that for most German stocks models with AR(1) component provide a better model fit than models with a risk premium of the type $\mu_{t}=\nu+\lambda \sqrt{h_{t}}$.

Thus, we refrain from applying the model of Duan (1995) and Härdle and Hafner (1999) directly, but rather modify the conditional mean to an $\mathrm{AR}(1)$ process. That is, under the data generating probability measure $P$ we assume the following $\mathrm{AR}(1)-\mathrm{TGARCH}(1,1)$ model

$$
\begin{aligned}
y_{t} & =\nu+\phi y_{t-1}+\sqrt{h_{t}} \xi_{t} \\
\xi_{t} & \sim i . i . d . N(0,1) \\
h_{t} & =\omega+\left(\left(\alpha+\alpha^{-} I_{\left(\xi_{t-1}<0\right)}\right) \xi_{t-1}^{2}+\beta\right) h_{t-1} .
\end{aligned}
$$

Under the measure $Q$, the model takes the following form:

$$
\begin{aligned}
y_{t} & =r+\sqrt{h_{t}} Z_{t} \\
Z_{t} & \sim i . i . d . N(0,1) \\
h_{t} & =\omega+\left(\left(\alpha+\alpha^{-} I_{\left(Z_{t-1}-\lambda_{t-1}<0\right)}\right)\left(Z_{t-1}-\lambda_{t-1}\right)^{2}+\beta\right) h_{t-1} \\
\lambda_{t} & =\frac{\nu+\phi y_{t-1}-r}{\sqrt{h_{t}}} .
\end{aligned}
$$

Note that unlike in the standard GARCH model, $h_{t}$ in (29) is not governed by central chisquare distributed innovations. This has an effect on the stationary variance of $y_{t}$, which is a determining factor for the global level of option prices. The following proposition provides the unconditional variance under $Q$ for the GARCH case. 
Proposition 3 For the system (27)-(30) with $\alpha^{-}=0$, the unconditional variance of $y_{t}$ under stationarity is finite if $\alpha\left(1+\phi^{2}\right)+\beta<1$, and

$$
\operatorname{Var}^{Q}\left[y_{t}\right]=\frac{\omega+\alpha(\nu-r(1-\phi))^{2}}{1-\alpha\left(1+\phi^{2}\right)-\beta} .
$$

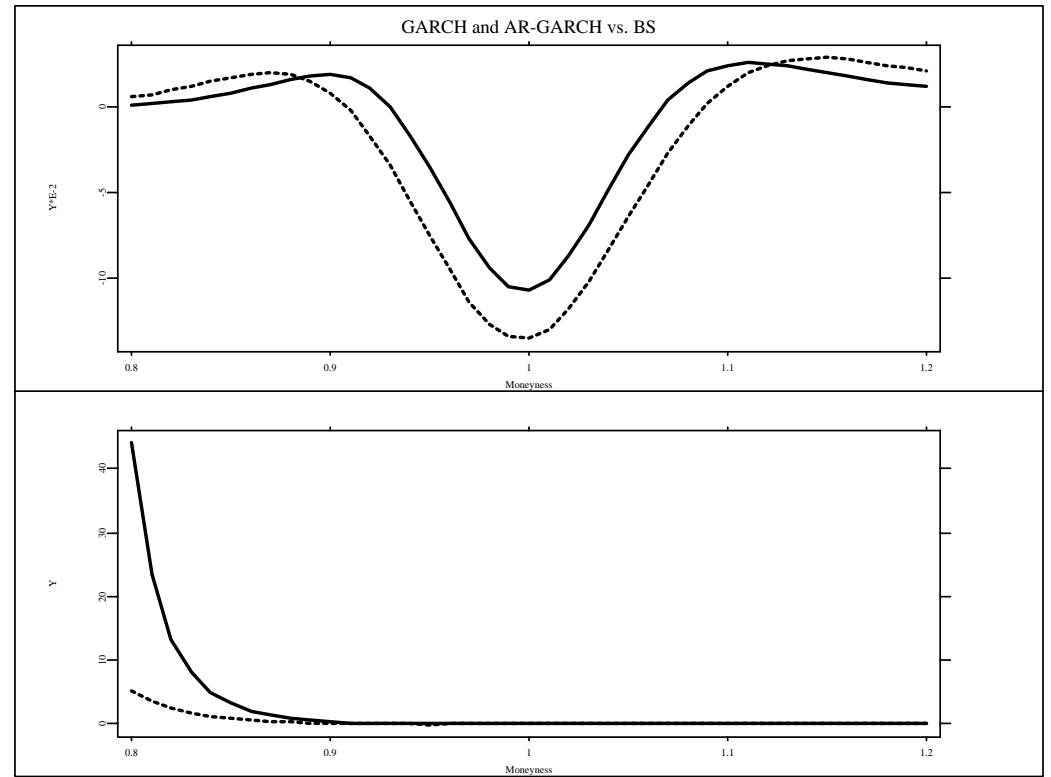

Figure 3: Upper panel: Difference between simulated GARCH option prices to Black and Scholes prices. GARCH parameters were set to $\alpha=0.09, \beta=0.9$. The $x$-axis represents the moneyness of the option. The dashed line incorporates AR(1) dynamics $(\phi=0.2)$, the solid one does not. Lower panel: the corresponding differences divided by the Black and Scholes price.

For the TGARCH case $\left(\alpha^{-} \neq 0\right), \operatorname{Var}^{Q}\left[y_{t}\right]$ is not tractable analytically but can be evaluated numerically. In the following, the unconditional variances are used for the computation of Black and Scholes option prices.

Since from (27) the discounted asset price process $(1+r)^{-t} S_{t}$ is a martingale, we can apply the no-arbitrage valuation methodology and obtain for the price of a European call option at time $t$,

$$
C_{t}=(1+r)^{-\tau} \mathrm{E}^{Q}\left[\max \left(S_{T}-K, 0\right) \mid \mathcal{F}_{t}\right] .
$$

The mean of the pay-off function in (31) is obtained via Monte Carlo simulation. The system (27) to (30) is generated 500,000 times. For the parameters in (29) we used typical parameter estimates obtained for the German stock return data: for the GARCH model $\omega=1 \mathrm{E}-06, \alpha=0.09, \beta=0.9$, and for the TGARCH model $\omega=1 \mathrm{E}-06, \alpha=0.03, \alpha^{-}=0.12$ and $\beta=0.9$. The $\operatorname{AR}(1)$ coefficient $\phi$ was set to 0.2 . Since the estimates $\hat{\nu}$ were found to be insignificant at the $5 \%$ level we set $r=\nu=0$ to evaluate (31). 


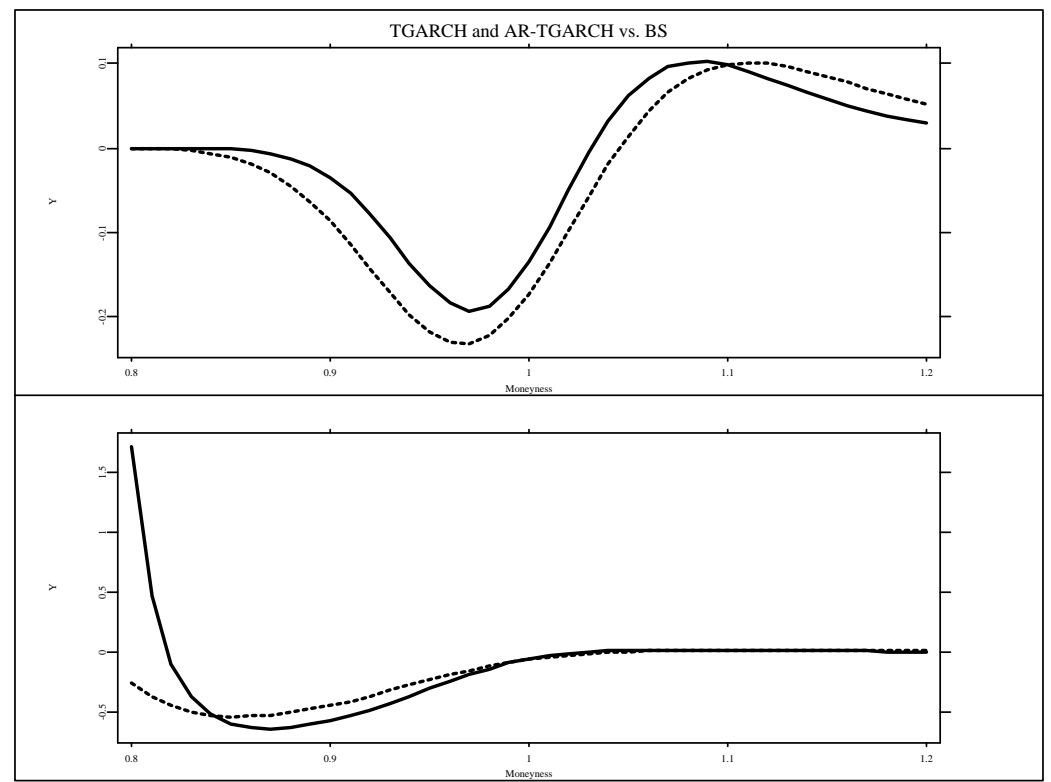

Figure 4: Upper panel: Difference between simulated TGARCH option prices to Black and Scholes prices. TGARCH parameters were set to $\alpha=0.03, \alpha^{-}=0.12, \beta=0.9$. The $x$-axis represents the moneyness of the option. The dashed line incorporates AR(1) dynamics $(\phi=0.2)$, the solid one does not. Lower panel: the corresponding differences divided by the Black and Scholes price.

Further, we used values for the time to maturity ( $\tau=30$ days) and moneyness $(S / K \in$ $[0.8,1.2])$ that resemble the parameters of traded options.

Since the standard benchmark model in option pricing is Black and Scholes (BS), we compare the simulated GARCH and TGARCH prices to BS prices. Our main focus is the difference between the impact of the existence and non-existence of a linear AR-component on option prices. We therefore estimated four models: AR(0)-GARCH, AR(1)-GARCH, $\mathrm{AR}(0)$-TGARCH, AR(1)-TGARCH, and compare the resulting option prices to BS prices.

Figure 3 displays in the upper plot the difference between AR(0)-GARCH prices (solid line) to BS prices as a function of moneyness. The dashed line is the corresponding difference for AR(1)-GARCH. The lower plot shows these differences divided by the BS price, thus giving some indication of the relative significance of the price differences.

Note first that for the GARCH differences to BS, we obtain the typical U-shape with out- and in-the-money options valued higher than BS and at-the-money options less.

Secondly (Figure 3), we investigate the difference between option prices with and without $\operatorname{AR}(1)$ dynamics. For options in a neighbourhood of at-the-money, prices are lower in the presence of $\mathrm{AR}(1)$ dynamics than without. The reverse is true for options sufficiently far inor out-of-the-money. Put differently, the U-shape and the deviation from Black \& Scholes prices become more pronounced under linear dynamics. Thus, for $\phi>0$, our results are in line with the results for continuous time models in Section 3 and Proposition 1.

Next, we are interested in the effects of the sign of $\phi$. Note from Proposition 2 with $\nu=r=0$ that the unconditional variance of $y_{t}$ does not depend on the sign of $\phi$. In 
fact, when replacing the positive value of $\phi$ by a negative one, one obtains the same picture as in Figure 3. Recall from Section 3 that in the Lo and Wang (1995) model negative autocorrelation induced by a univariate trending OU-process and positive autocorrelation obtained for a bivariate one have inverse implications for the estimated level of volatility and, hence, for option prices. Thus, concerning the sign of the autoregression parameter, the implications for option prices in discrete time differ from the Lo and Wang continuous time model.

Note from the lower part of Figure 3 that the relative significance of the option price differences is highest for far out-of-the-money options. This corresponds to the high elasticity of far out-of-the-money options to changes in volatility, which was shown in Figure 2 for the Black \& Scholes model.

Qualitatively similar results are obtained for the TGARCH model, see Figure 4 . The main difference is that the curves are shifted to the left, reflecting the leverage effect. For example, options out-of-the-money do not benefit from the leverage effect. This is because for the out-of-the-money option to be of positive value at maturity, a sequence of positive innovations is required. Positive innovations, however, do not have an increasing effect on volatility in the presence of a leverage effect. So there is a negative effect of positive innovations by reducing volatility. Of course, when considering the less realistic case $\alpha^{-}<0$, which may be called an inverted leverage effect, the skew of the curves in Figure 4 is mirrored: out-of-the-money options become more, in-the-money options less expensive.

\section{Linear dynamics, GARCH, and conditional leptokur- tosis}

Conditional leptokurtosis is a well-documented empirical effect for various financial time series. In most cases, the inclusion of an ARCH-type volatility does not fully account for the observed leptokurtosis of returns. QML estimation remains consistent if the innovation distribution is not normal, as was shown by Bollerslev and Wooldridge (1992). The efficiency loss was studied by Engle and Gonzalez-Rivera (1991). Their results suggest that e.g. for a Student-t distribution with 5 degrees of freedom and a $\operatorname{GARCH}(1,1)$ process the asymptotic variance of QML estimates may be more than double the variance of ML estimates with correct specification.

There have been many approaches to model a fat-tailed innovation distribution directly, the most popular being the Student-t first used by Bollerslev (1987). Recently, Platen (1999) has established a general economic model which motivates the prevalence of Studentt distributions for stock returns. Duan (1999) does not consider Student-t distributions, because he needs a well-defined moment generating function, which the Student-t fails to have. Rather, as Nelson (1991) he employs the generalized error distribution (GED) that also exhibits fat tails and includes the normal distribution as a special case. However, in his study of stock returns Nelson concluded that the tail-thickness of the GED may not suffice to adequately model extremal returns. In our case, we are able to use the Student-t since we defined returns as $y_{t}=\left(S_{t}-S_{t-1}\right) / S_{t-1}$ rather than $y_{t}=\log \left(S_{t} / S_{t-1}\right)$.

The basic idea of Duan's (1999) extension of his GARCH option pricing model is a transformation of the fat-tailed innovation to a standard normal one, under which his locally risk neutral valuation relationship remains valid. In our case with simple returns and the Student-t distribution, the model under the data generating probability measure $P$ generalizes from (16), (17) and (18) as

$$
\begin{aligned}
y_{t} & =\mu_{t}+\sqrt{h_{t}} \xi_{t} \\
\xi_{t} & \sim i . i . d . t_{v}(0,1) \\
h_{t} & =f\left(h_{s}, \xi_{s} ;-\infty<s<t ; \theta\right) .
\end{aligned}
$$


where $t_{v}(0,1)$ is the standardized Student-t distribution with $v$ degrees of freedom. Denote by $G_{v}$ the Student-t distribution function with $v$ degrees of freedom, standardized to have variance one. ${ }^{2}$ Then, $\xi_{t}$ can be transformed to a standard normal random variable by

$$
\Psi_{v}\left(\xi_{t}\right)=\Phi^{-1}\left[G_{v}\left(\xi_{t}\right)\right]
$$

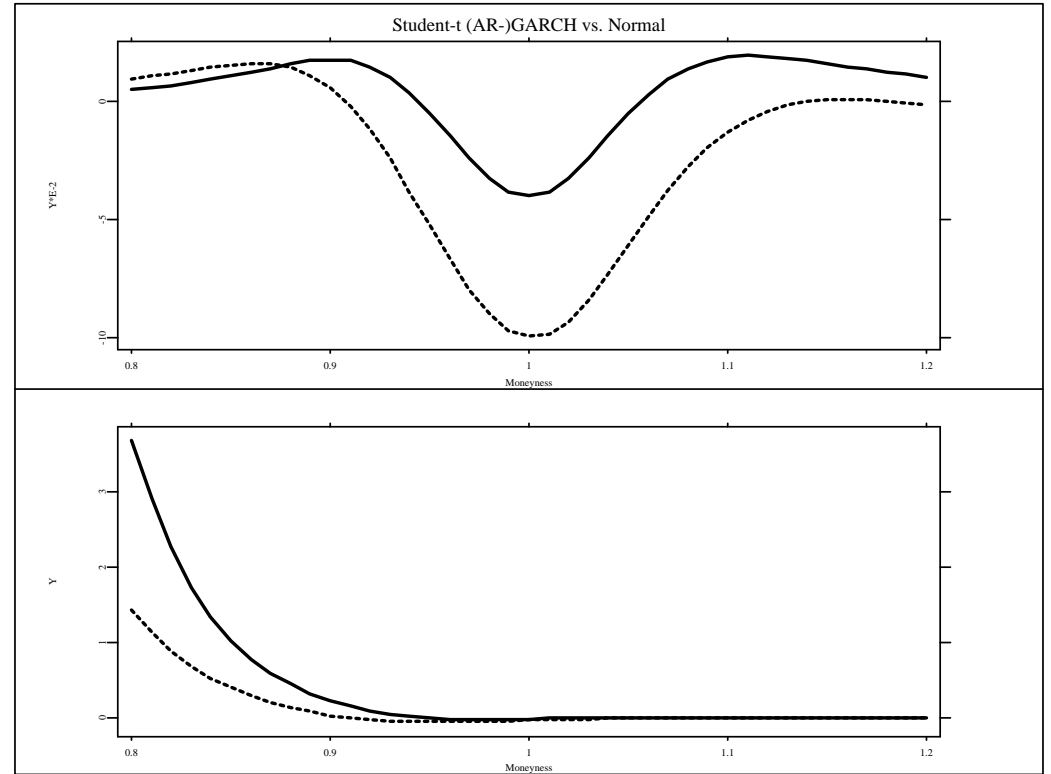

Figure 5: Upper panel: Difference between simulated GARCH option prices with Student-t innovations to GARCH prices with normal innovations. GARCH parameters were set to $\alpha=0.09, \beta=0.9$ and the Student- $t$ degrees of freedom to $v=6.4$. The $x$-axis represents the moneyness $S_{t} / K$ of the option. The dashed line incorporates AR(1) dynamics with $\phi=0.2$, the solid one does not. Lower panel: the corresponding differences divided by the (AR-) GARCH price with normal innovations.

Under the equivalent martingale measure $Q$, the model writes as

$$
\begin{aligned}
y_{t} & =\mu_{t}+\sqrt{h_{t}} \Psi_{v}^{-1}\left(Z_{t}-\lambda_{t}\right) \\
Z_{t} & \sim i . i . d . N(0,1) \\
h_{t} & =f\left(h_{s}, \xi_{s} ;-\infty<s<t ; \theta\right) \\
\xi_{t} & =\Psi_{v}^{-1}\left(Z_{t}-\lambda_{t}\right),
\end{aligned}
$$

where $\lambda_{t}$ is the solution to

$$
\mathrm{E}^{Q}\left[\Psi_{v}^{-1}\left(Z_{t}-\lambda_{t}\right) \mid \mathcal{F}_{t-1}\right]=\frac{r-\mu_{t}}{\sqrt{h_{t}}}
$$

\footnotetext{
${ }^{2}$ In many statistical programming packages the Student-t distribution function is available. In Gauss, one minus this function is given by cdftc () . To obtain the corresponding standardized distribution function, use $G_{v}(x)=1-c d f t c\left(x \sqrt{\frac{v-2}{v}}\right)$. To compute the inverse of $G$, use the Gauss function cdftci as $G_{v}^{-1}(x)=$ $\operatorname{cdftci}(x) \sqrt{\frac{v}{v-2}}$.
} 


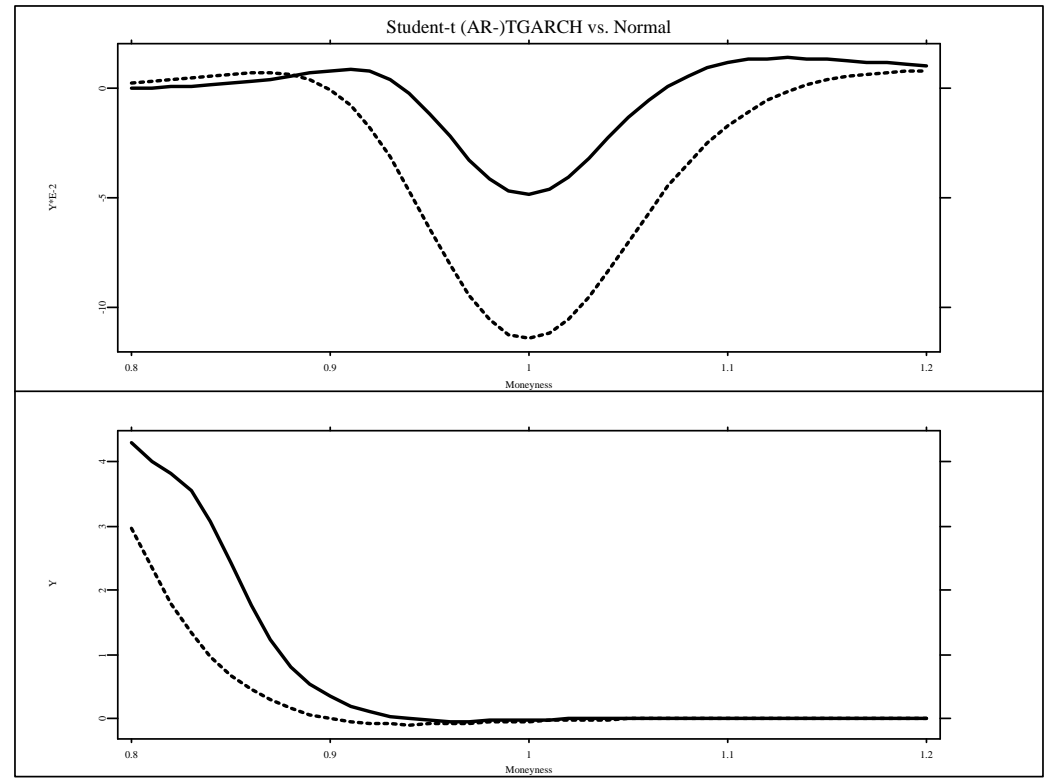

Figure 6: Upper panel: Difference between simulated TGARCH option prices with Student-t innovations to GARCH prices with normal innovations. TGARCH parameters were set to $\alpha=0.03, \alpha^{-}=0.12, \beta=0.9$ and the Student-t degrees of freedom to $v=6.4$. The $x$-axis represents the moneyness of the option. The dashed line incorporates AR(1) dynamics with $\phi=0.2$, the solid one does not. Lower panel: the corresponding differences divided by the (AR-) GARCH price with normal innovations.

The conditional expectation on the left hand side of (39) is a function of $\lambda_{t}$ and can be calculated numerically. As in the previous sections, we set $\mu_{t}=\nu+\phi y_{t-1}$ and $h_{t}$ to be the TGARCH process given in (26).

To investigate the effects of conditional Student-t innovations on option prices in the current model framework, we show the differences of option prices generated under normal innovations and corresponding prices under Student-t innovations. For the dynamics of the return process, we continue to consider the GARCH and TGARCH type volatility processes as well as autoregressive, respectively no autoregressive, dynamics in the conditional mean. The parameters were set at the same values as in the previous section. The degrees of freedom for Student-t distribution were fixed at $v=6.4$, which corresponds to typical estimates for the German stock returns, see Table 1.

Figure 5 shows the differences of option prices under GARCH and normality to corresponding option prices under GARCH and Student-t innovations. The dashed (solid) line represents the situation of (no) autoregressive dynamics. The results can be summarized as follows: Both effects found in the previous section, i.e., the smile-formed shape of the option price difference caused by GARCH and the increase of the smile caused by AR dynamics become even more pronounced under conditional leptokurtosis. In relative terms, this is particularly significant for out-of-the-money options. A similar picture arises for the TGARCH case in Figure 6.

Finally, Table 5 reports the percentage differences of simulated option prices using the 


\begin{tabular}{|c|c|c|c|c|c|c|c|c|}
\hline & \multicolumn{4}{|c|}{$\overline{\xi_{t} \sim N(0,1)}$} & \multicolumn{4}{|c|}{$\overline{\xi_{t} \sim t_{v}(0,1)}$} \\
\hline & \multicolumn{2}{|c|}{ GARCH } & \multicolumn{2}{|c|}{ TGARCH } & \multicolumn{2}{|c|}{ GARCH } & \multicolumn{2}{|c|}{ TGARCH } \\
\hline Stock & $S_{t} / K=0.8$ & 0.9 & 0.8 & 0.9 & 0.8 & 0.9 & 0.8 & $\overline{0.9}$ \\
\hline$\overline{\mathrm{ALLI}}$ & 21.18 & 11.03 & 46.39 & 14.74 & 1.74 & 1.87 & 3.21 & 2.37 \\
\hline BASF & -.83 & .180 & -.24 & .51 & .35 & .64 & .61 & 68 \\
\hline BAYE & -3.40 & -1.25 & -.57 & -.08 & -.87 & -.40 & .74 & .09 \\
\hline BAYH & -7.90 & -.67 & -3.27 & .79 & -3.59 & -.81 & .38 & .73 \\
\hline BAYV & 6.24 & 5.00 & 4.81 & 4.82 & .13 & 1.33 & 2.42 & 1.51 \\
\hline BMW & -2.07 & 2.09 & -2.09 & 2.32 & -12.61 & -4.06 & -10.48 & -3.32 \\
\hline COMM & .54 & .65 & 1.09 & .88 & 43 & .48 & 1.23 & .93 \\
\hline DAIM & -2.24 & .38 & .75 & 1.08 & -3.57 & -.54 & -2.16 & .08 \\
\hline DEUT & -2.38 & -.11 & 12.81 & 2.16 & -0.13 & .64 & -1.32 & .58 \\
\hline DEGU & .50 & .41 & 1.21 & .49 & -2.09 & -.50 & -1.27 & -.40 \\
\hline DRES & 2.96 & 1.96 & 5.11 & 3.07 & -3.96 & -2.01 & -6.17 & -2.10 \\
\hline HENK & -4.06 & -1.41 & -3.38 & -1.09 & .66 & .74 & .05 & .56 \\
\hline HOEC & -6.60 & -.41 & -5.30 & .23 & -6.43 & -1.30 & -6.02 & -.69 \\
\hline KARS & -6.97 & -.92 & -5.11 & -.55 & -9.95 & -1.14 & 8.54 & 1.19 \\
\hline LIND & -32.84 & -4.70 & -25.89 & -1.72 & -19.04 & -5.60 & -16.34 & -4.75 \\
\hline MAN & -2.63 & .51 & 5.06 & 1.59 & -5.94 & -.89 & -8.84 & -1.06 \\
\hline MANN & 2.77 & 1.11 & 7.29 & 1.83 & .07 & 1.27 & 3.96 & 2.34 \\
\hline MUEN & -26.14 & -3.64 & -27.61 & -2.53 & -10.58 & -6.49 & -18.80 & -9.97 \\
\hline PREU & 15.38 & 7.65 & 11.92 & 7.47 & -6.13 & -1.00 & -7.41 & -.79 \\
\hline RWE & 27.96 & 8.91 & 36.01 & 10.3 & -1.21 & .08 & -4.44 & -.29 \\
\hline SCHE & -7.40 & -.45 & -6.92 & -.40 & -4.03 & -1.15 & -5.88 & -1.59 \\
\hline SIEM & 3.25 & .23 & -3.37 & -1.12 & 5.43 & -.34 & -3.89 & -2.76 \\
\hline THYS & 1.40 & .15 & 1.27 & -.08 & 1.12 & .47 & 1.02 & .40 \\
\hline VEBA & 6.37 & 2.70 & 2.71 & 1.28 & 1.14 & 1.18 & -.55 & .31 \\
\hline VIAG & -10.34 & -2.53 & -10.74 & -3.03 & -1.73 & .39 & 6.43 & .93 \\
\hline VOLK & -.90 & .06 & 2.44 & 0.67 & -.11 & .44 & -.15 & .67 \\
\hline
\end{tabular}

Table 5: Percentage differences of simulated option prices using the parameter estimates for the German stock price series.

parameter estimates for the German stock price series. Large percentage differences can be found for Allianz, Linde, Münchner Rück, Preussag, RWE and Viag, which corresponds to large discrepancies in the volatility estimates reported in Tables 3 and 4 . The largest percentage difference, $46.39 \%$, occurs for Allianz with a conditionally normal TGARCH model.

\section{Conclusions and Outlook}

Modelling volatility with or without linear AR(1) dynamics in the conditional mean yields volatility estimates of some German stocks that differ substantially. As option prices crucially depend on volatility estimates, the presence of autoregressive dynamics may affect option prices strongly if the elasticity of option prices with respect to volatility is high. To analyse the qualitative and quantitative dimension of this effect, we have shown that in a continuous time framework the presence of a trend reversion component for the log price of the underlying stock increases the deviation from Black-Scholes prices caused by stochastic volatility. The analytic results for our continuous-time model provide useful insights, but the model is rather restricted: it can only display negative autocorrelation of returns and orthogonality of the two risk sources (i.e., no leverage effect). Therefore, we further con- 
sidered a discrete-time framework with $\mathrm{AR}(1)$ conditional mean and GARCH conditional variance. To account for stylized facts of stock returns, we considered the leverage effect and conditional leptokurtosis. Using Monte Carlo simulation, we evaluate option prices under the risk-neutral pricing measure. Our results may be summarized as follows:

1. GARCH effects imply a smile-shaped difference of option prices to Black and Scholes prices, viewed as a function of the moneyness.

2. The leverage effect, captured by the TGARCH model, skews this smile with out-ofthe-money options becoming less expensive.

3. The inclusion of linear autoregressive dynamics, $\operatorname{AR}(1)$, affects option prices. The deviation of option prices from the BS model tends to increase in the presence of autoregressive dynamics. This deviation only depends on the size, not on the sign of the autoregressive parameter.

4. Conditional leptokurtosis imposes another smile: the smile-formed shape of the option price difference caused by GARCH and the increase of the smile caused by AR dynamics become even more pronounced. Under TGARCH, the difference of option prices assuming conditional normality versus Student-t remains symmetric with respect to the moneyness, but is again increased in the presence of AR effects.

As weakly autoregressive processes such as an $\operatorname{AR}(1)$ process with $\phi=0.1$ are empirically indistinguishable from a low order moving average process, one may ask how option prices are affected when using MA instead of AR models. In addition, the specification of the conditional mean equation may be generalized to account for weekday effects which can be found for German stock market returns, see e.g. Herwartz (1999). Finally, using stochastic volatility models with separate stochastic innovations in the volatility process may give some different results than for GARCH-type models, although we conjecture that our qualitative results remain unchanged.

\section{Appendix A}

Proof of Proposition 1: From Drost and Werker (1996), we have the moments

$$
\begin{aligned}
\mathrm{E}\left[\sigma_{t}^{2}\right] & =v \\
\mathrm{E}\left[\sigma_{t}^{2} \sigma_{s}^{2}\right] & =v^{2}+\frac{v^{2} \delta^{2}}{2\left(\theta-\delta^{2} / 2\right)} e^{-|t-s| \theta} .
\end{aligned}
$$

Using Fubini's theorem, we obtain

$$
\mathrm{E}\left[\bar{V}_{\tau}\right]=\frac{1}{\tau} \int_{0}^{\tau} \mathrm{E}\left[\sigma_{t}^{2}\right] d t=v
$$

and, for $t>s$,

$$
\begin{aligned}
\mathrm{E}\left[\bar{V}_{\tau}^{2}\right] & =\frac{2}{\tau^{2}} \int_{0}^{\tau} \int_{0}^{t} \mathrm{E}\left[\sigma_{t}^{2} \sigma_{s}^{2}\right] d s d t \\
& =v^{2}+\frac{v^{2} \delta^{2}}{\left(\theta-\delta^{2} / 2\right)} \frac{\theta \tau+e^{-\theta \tau}-1}{\theta^{2} \tau^{2}} .
\end{aligned}
$$

Proof of Proposition 2: We have

$$
\frac{\partial B}{\partial \delta^{2}}=\frac{\theta}{4\left(\theta-\delta^{2} / 2\right)^{2}} \frac{\theta \tau+e^{-\theta \tau}-1}{\theta^{2} \tau}>0,
$$


and

$$
\frac{\partial B}{\partial \theta}=\frac{\delta^{2}}{4} \frac{\theta \tau \delta^{2} / 2\left(1+e^{-\theta \tau}\right)+\left(3 \theta-\delta^{2}\right)\left(1-e^{-\theta \tau}\right)-\theta^{2} \tau\left(2+e^{-\theta \tau}\right)}{\theta^{3} \tau^{2}\left(\theta-\delta^{2} / 2\right)^{2}},
$$

which is negative under the fourth moments condition $\delta^{2}<2 \theta$. Furthermore, we have

$$
\frac{\partial A}{\partial \sqrt{v}}=\psi\left(d_{1}\right)\left(d_{1}^{4}-2 \sqrt{v \tau} d_{1}^{3}+(v \tau-2) d_{1}^{2}+2 \sqrt{v \tau} d_{1}-(1+v \tau)\right) .
$$

Since $\psi\left(d_{1}\right)>0$, the sign of $\frac{\partial A}{\partial \sqrt{v}}$ is determined by the sign of the polynomial in $d_{1}$,

$$
d_{1}^{4}-2 \sqrt{v \tau} d_{1}^{3}+(v \tau-2) d_{1}^{2}+2 \sqrt{v \tau} d_{1}-(1+v \tau),
$$

which is negative at $d_{1}=0$ and positive for large absolute values of $d_{1}$. The zeros of (42) are

$$
d_{1}=\frac{\sqrt{v \tau}}{2} \pm \frac{1}{2} \sqrt{v \tau+4(1 \pm \sqrt{2+v \tau})}
$$

or, equivalently,

$$
\log \left(S_{t} / K\right)= \pm \frac{1}{2} \sqrt{v \tau+4(1 \pm \sqrt{2+v \tau})}-r \tau .
$$

As long as $v \tau<4+\sqrt{32}$, there are only two real zeros in $d_{1}$, a positive and a negative one. In terms of the moneyness $S_{t} / K$, as long as $r \tau<1 / 2 \sqrt{v \tau+4(1+\sqrt{2+v \tau})}$ there is one positive and one negative zero in $\log \left(S_{t} / K\right)$. This implies that at the money (values in a neighbourhood of $\left.\log \left(S_{t} / K\right)=0\right)$ the derivative is negative. On the other hand, for $\log \left(S_{t} / K\right)>\frac{1}{2} \sqrt{v \tau+4(1+\sqrt{2+v \tau})}-r \tau$ and for $\log \left(S_{t} / K\right)<-\frac{1}{2} \sqrt{v \tau+4(1+\sqrt{2+v \tau})}-$ $r \tau$ the derivative is positive. Thus, at-the-money options become less, far in- and out-ofthe-money options more valuable in the presence of a trending OU-process.

\section{Proof of Proposition 3:}

Because of $\mathrm{E}^{Q}\left[y_{t}\right]=r, \mathrm{E}^{Q}\left[y_{t}^{2}\right]=r^{2}+\mathrm{E}^{Q}\left[h_{t}\right]$ and $\mathrm{E}^{Q}\left[h_{t} Z_{t} \lambda_{t}\right]=0$, we can write

$$
\begin{aligned}
\mathrm{E}^{Q}\left[h_{t}\right] & =\omega+\alpha \mathrm{E}^{Q}\left[h_{t-1}\right]+\alpha \mathrm{E}^{Q}\left[\left(\nu-r+\phi y_{t-2}\right)^{2}\right]+\beta \mathrm{E}^{Q}\left[h_{t-1}\right] \\
& =\omega+(\alpha+\beta) \mathrm{E}^{Q}\left[h_{t-1}\right]+\alpha(\nu-r(1-\phi))^{2}+\alpha \phi^{2} \mathrm{E}^{Q}\left[h_{t-2}\right] .
\end{aligned}
$$

Under stationarity, the unconditional expectations are independent of $t$ and the result is obtained.

\section{Appendix B}

Suppose that a continuous time stochastic process $Y_{t}$ is observed at regular time intervals of length $h>0$ and call this process $Y_{(h) t}, t \in h \mathbb{N}$. The process $Y_{t}$ is called a GARCH diffusion if for each $h>0$ the process $Y_{(h) t}, t \in h \mathbb{N}$ is a weak GARCH process in the sense of Drost and Nijman (1993) with

$$
\sigma_{(h) t}^{2}=\omega_{h}+\alpha_{h} Y_{(h) t-h}^{2}+\beta_{h} \sigma_{(h) t-h}^{2}
$$

being the best linear predictor of $Y_{(h) t}^{2}$ in terms of $1, \sigma_{(h) 0}^{2}$ and lagged values of $Y_{(h) t}$ and $Y_{(h) t}^{2}$. 


\begin{tabular}{c|rrrrrrr}
\hline \hline & \multicolumn{7}{|c}{$\phi$} \\
& -0.5 & -0.2 & -0.1 & 0 & 0.1 & 0.2 & 0.5 \\
\hline$\alpha_{1}$ & 0.255 & 0.112 & 0.103 & 0.100 & 0.103 & 0.112 & 0.260 \\
Ste $\left(\alpha_{1}\right)$ & 1.540 & 0.636 & 0.575 & 0.561 & 0.582 & 0.649 & 1.571 \\
\hline$\beta_{1}$ & 0.532 & 0.818 & 0.834 & 0.839 & 0.834 & 0.817 & 0.523 \\
Ste $\left(\beta_{1}\right)$ & 4.460 & 1.356 & 1.110 & 1.050 & 1.172 & 1.420 & 4.430 \\
\hline$\omega_{1}$ & 0.290 & 0.074 & 0.064 & 0.061 & 0.064 & 0.075 & 0.296 \\
Ste $\left(\omega_{1}\right)$ & 4.402 & 1.027 & 0.805 & 0.750 & 0.867 & 1.090 & 4.370 \\
\hline \hline$\theta$ & 0.261 & 0.074 & 0.066 & 0.064 & 0.066 & 0.075 & 0.265 \\
Ste $(\theta)$ & 4.956 & 1.235 & 0.963 & 0.893 & 1.179 & 1.384 & 4.916 \\
\hline $100 \delta^{2}$ & 11.263 & 0.106 & 0.034 & 0.020 & 0.106 & 0.156 & 11.387 \\
Ste $\left(\delta^{2}\right)$ & 7.715 & 0.572 & 0.214 & 0.111 & 0.879 & 1.091 & 7.557 \\
\hline \hline$d B(\tau=0.01)$ & 0.321 & 0.021 & 0.004 & 0.000 & 0.005 & 0.022 & 0.329 \\
$d B(\tau=0.1)$ & 3.811 & 0.213 & 0.045 & 0.000 & 0.055 & 0.227 & 3.902 \\
$d B(\tau=1)$ & 37.988 & 2.095 & 0.440 & 0.000 & 0.548 & 2.230 & 38.890 \\
$d B(\tau=10)$ & 317.792 & 17.502 & 3.679 & 0.000 & 4.581 & 18.635 & 325.335 \\
\hline \hline
\end{tabular}

Table 6: Deviations of discrete and implied continuous time parameter estimates when the true DGP contains an autoregressive parameter $\phi$. The volatility parameters of the simulated $D G P$ are $\alpha_{1}=0.1, \beta_{1}=0.85$, and $\omega_{1}=0.05$. Ste denotes the standard error of 2000 Monte Carlo replications, measured in $E-03 . d B$ is the total differential of $B$ given in (15) with respect to the parameter changes of $\theta$ and $\delta$, measured in $E-02$.

As shown by Drost and Werker (1996), the mappings between the discrete and continuous time parameters in (43) and (10), respectively, are given by

$$
\begin{aligned}
\theta & =-\frac{1}{h} \log \left(\alpha_{h}+\beta_{h}\right) \\
\varpi & =\frac{1}{h} \omega_{h} \\
\delta^{2} & =-\frac{1}{h} \frac{2 \log ^{3}\left(\alpha_{h}+\beta_{h}\right)}{\frac{1-\left(\alpha_{h}+\beta_{h}\right)^{2}\left(1-\beta_{h}^{2}\right)}{\alpha_{h}\left(1-\beta_{h}\left(\alpha_{h}+\beta_{h}\right)\right)}+6 \log \left(\alpha_{h}+\beta_{h}\right)+2 \log ^{2}\left(\alpha_{h}+\beta_{h}\right)+4\left(1-\alpha_{h}-\beta_{h}\right)}
\end{aligned}
$$

Now, for a given time interval $h$, which w.l.o.G. we set to one, we can estimate the GARCH parameters $\omega_{1}, \alpha_{1}$ and $\beta_{1}$ and obtain the corresponding continuous time parameters $\theta, \varpi$ and $\delta$ through the equations (44) to (46). We do that by letting the true data generating process contain an autoregressive component but estimating a (misspecified) AR(0)-GARCH model. This conveys information about the change of parameter estimates.

The parameter estimates and corresponding standard errors of 2000 Monte Carlo replications are given in Table 6 . Denote the difference of the parameter estimates under nonzero $\phi$ to the corresponding estimates under $\phi=0$ by $d \varpi, d \delta^{2}$ and $d \theta$. We are interested in the sign of the total differential

$$
d B=\frac{\partial B}{\partial \delta^{2}} d \delta^{2}+\frac{\partial B}{\partial \theta} d \theta,
$$

where $\partial B / \partial \delta^{2}$ and $\partial B / \partial \theta$ are given in (40) and (41). For selected values of $\tau, d B$ always turned out to be positive (see Table 6$)$, indicating that the negative effect through $(\partial B / \partial \theta) d \theta$ is outweighed by the positive effect of $\left(\partial B / \partial \delta^{2}\right) d \delta^{2}$. 


\section{References}

Black, F. (1976), Studies in stock price volatility changes, Proceedings of the 1976 Meeting of the Business and Economics Statistics Section, American Statistical Association: $177-181$.

Black, F. and M. Scholes (1973), The pricing of options and corporate liabilities, Journal of Political Economy, 81, 637-659.

Bollerslev, T. (1986), Generalized autoregressive conditional heteroskedasticity, Journal of Econometrics 31: 307-327.

Bollerslev, T. (1987), A conditional heteroskedastic time series model for speculative prices and rates of returns, Review of Economics and Statistics, 69, 542-547.

Bollerslev, T. and J.M. Wooldridge (1992), Quasi maximum likelihood estimation and inference in dynamic models with time varying covariances Econometric Reviews 11: $143-172$.

Campbell, J., A. Lo and A. MacKinlay (1997), The Econometrics of Financial Markets, Princeton University Press, Princeton, New Jersey.

Drost, F. C. and T. E. Nijman (1993), Temporal aggregation of GARCH processes, Econometrica 61: 909-927.

Drost, F. C. and B. J. M. Werker (1996), Closing the GARCH gap: continuous time GARCH modeling, Journal of Econometrics 74: 31-57.

Duan, J.-C. (1995), The GARCH option pricing model, Mathematical Finance, 5: 13-32.

Duan, J.-C. (1996), A unified theory of option pricing under stochastic volatility - from GARCH to diffusion, Department of Finance, Hong Kong University of Science and Technology.

Duan, J.-C. (1999), Conditionally fat-tailed distributions and the volatility smile in options, Working paper, Department of Finance, Hong Kong University of Science and Technology.

Engle, R.F. (1982), Autoregressive conditional heteroskedasticity with estimates of the variance of U.K. inflation, Econometrica, 50, 987-1008.

Engle, R.F. and G. Gonzalez-Rivera (1991), Semiparametric ARCH models, Journal of Business 83 Economic Statistics 9:345-359.

Engle, R.F., D.M. Lilien and R.P. Robins (1987), Estimating time varying risk premia in the term structure: The ARCH-M model, Econometrica 55: 391-407.

Föllmer, H., M. Schweizer (1991), Hedging of Contingent Claims under Incomplete Information, in: Applied Stochastic Analysis, ed. by M.H.A.Davis and R.J.Elliot, Gordon and Breach, London, 389-414.

Föllmer, H., D. Sondermann (1986), Hedging of Non-redundant Contingent Claims, in: Hildenbrand, W., Mas-Colell, A. (eds.), Contributions to Mathematical Economics, Amsterdam, North Holland, 205-223.

Glosten, L., R. Jagannathan and D. Runkle (1993), Relationship between the expected value and the volatility of the nominal excess return on stocks, Journal of Finance 48: 1779-1801. 
Härdle, W. and C.M. Hafner (1999), Discrete time option pricing with flexible volatility estimation, Finance and Stochastics, forthcoming.

Hafner, C.M. and H. Herwartz (1998), Structural analysis of portfolio risk using beta impulse response functions, Statistica Neerlandica 52: 336-355.

Hafner, C.M. and H. Herwartz (1999), Testing for linear autoregressive dynamics under heteroskedasticiy, SFB 373 Discussion Paper 07, Humboldt-Universität zu Berlin.

Herwartz, H. (1998), Testing periodicity in time series models - A recommendation of bootstrap methods, Computational Statistics 13: 283-300.

Herwartz, H. (1999), Weekday dependence of German stock market returns, Applied Stochastic Models in Business and Industry, forthcoming.

Hofmann, N., E. Platen and M. Schweizer (1992), Option pricing under incompleteness and stochastic volatility, Mathematical Finance 2: 153-187.

Hull, J. and A. White (1987), The pricing of options on assets with stochastic volatilities, Journal of Finance 42: 281-299.

Lo, A. and J. Wang (1995), Implementing option pricing models when asset returns are predictable, Journal of Finance 50: 87-129.

Nelson, D. B. (1990), ARCH models as diffusion approximations, Journal of Econometrics 45: $7-38$.

Nelson, D. B. (1991), Conditional heteroskedasticity in asset returns: a new approach, Econometrica 59: 347-370.

Platen, E. (1999), A financial market model, Quantitative Finance Research Group, Research paper 9, University of Technology, Sydney, Australia.

White, H. (1980), A heteroskedasticity consistent covariance matrix estimator and a direct test for heteroskedasticity, Econometrica 48: 817-838

Zakoian, J.M. (1994), Threshold heteroskedastic functions, Journal of Economic Dynamics and Control 18: 931-955. 